\title{
Status of the TUS space experiment preparation
}

\section{Leonid Tkachev ${ }^{1}$}

Joint Institute for Nuclear Research

Dubna, Moscow region, 141980 Russia

E-mail: Leonid.Tkatchev@cern.ch

\section{G. Garipov, B. Khrenov, N. Kalmykov, P. Klimov, M. Panasyuk, S. Sharakin, A.} Shirokov, I. Yashin

Skobeltsyn Institute of Nuclear Physics, Moscow State University, Moscow, Russia

\section{S. Biktemerova, A. Grinyuk, V. Grebenyuk, D. Naumov, S. Porokhovoy, B.} Sabirov, M. Slunecka, A. Tkachenko

Joint Institute for Nuclear Research

\section{A.Puchkov, O. Saprykin}

Space Regatta Consortium, Korolev, Moscow region, Russia

I. Park, J. Lee, S. Nam,, G. Na

Physics Department, EWHA Woman University, Seoul, Korea

\section{O. Martinez, H. Salazar}

University of Puebla, Puebla, Puebla, Mexico

\section{Villasenor}

University of Michiocan, Morelia, Michiocan, Mexico

The TUS space project for investigation of Ultra High Energy Cosmic Rays (UHECR) by the measurement of Extensive Atmospheric Shower fluorescent radiation is in the construction stage. The main goal of the TUS mission is to search for cosmic ray particles beyond the GZK energy limit. The observation of the full sky for primary particle arrival directions is an advantage of the TUS project in comparison to the ground based experiments. The TUS experience of UHECR study from the space will be of importance for future space detectors like the JEM-EUSO mission. The TUS detector operation could be considered as a "pathfinder" for the JEM-EUSO mission including the JEM-EUSO UV sensor test. The technological TUS prototype is produced and their tests are in progress. The flight detector has to be produced in 2010-2011. The mission is planned for operation at the end of 2011 at the dedicated "Mikhail Lomonosov" satellite.

35th International Conference of High Energy Physics (ICHEP2010)

Paris,France

July 22-28, 2010

$1 \quad$ Speaker 


\section{Introduction}

The TUS project task is an experimental study of the UHECR. The fluorescent and Cherenkov radiation of Extensive Air Showers (EAS) generated by UHECR particles will be detected at night side of the Earth atmosphere from the space platform at heights $400-500 \mathrm{~km}$. It will make possible to measure the $\mathrm{CR}$ spectrum, composition and angular distribution at $\mathrm{E}>$ $7 \cdot 10^{19} \mathrm{eV}$ beyond the GZK energy limit. There are two main parts of this detector: a modular Fresnel mirror and a matrix of PMTs with corresponding DAQ electronics. The SINP MSU (main investigator), JINR and Consortium "Space Regatta" together with several Korean and Mexican Universities are collaborating in the TUS detector preparation. The TUS mission is now planned for operation at the dedicated "Mikhail Lomonosov" satellite shown in Fig.1.

Main TUS parameters: mass $<60 \mathrm{~kg}$, power consumption $\sim 65 \mathrm{~W}$, data rate 200 Mbytes/day (1 EAS event contains $\sim 80$ Kbytes), Field-of-View \pm 4.5 degree, number of pixels 16x16 (Hamamatsu type R1463 PMT: $13 \mathrm{~mm}$ tube diameter, multi-alcali cathode, UV glass window), pixel FOV $\sim 10 \mathrm{mrad}$, Fresnel mirror area $1.8 \mathrm{~m}^{2}$, focal distance $1.5 \mathrm{~m}$.

Photo detector and electronics consists of 256 pixels with the time resolution $0,8 \mu$ s and the spatial resolution $5 \times 5 \mathrm{~km}$ (for the orbit height of $500 \mathrm{~km}$ ). The digital integrators allow to use the same photo detector to study different phenomena in the atmosphere in wide time interval: $\sim 100 \mu \mathrm{s}$ (EAS) and $1 \mathrm{~ms}-1 \mathrm{~s}$ (transient luminous events, TLE). That principle was tested during 2 years of "Universitetsky-Tatiana" mission [1].

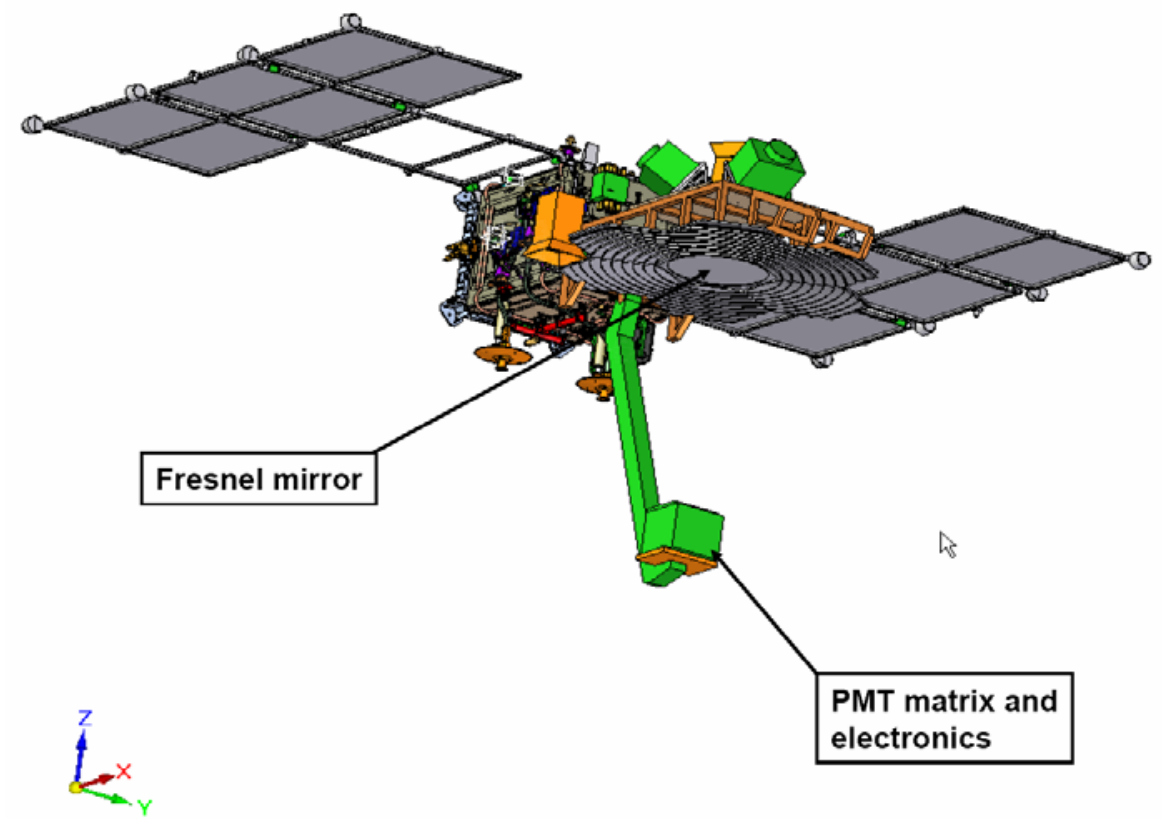

Fig.1. The TUS detector at the "Mikhail Lomonosov" satellite. 
In the TUS photo detector box the pinhole camera is added for study of TLE. The pinhole camera consists of multianode PMT and a hole at the focal distance from the PMT cathode. In design of the camera the multi-anode PMT of JEM-EUSO type is used [2]. The JEM-EUSO UV sensor will be tested during the TUS data taking for atmospheric TLE measurements by pinhole camera.

\section{Status of the TUS preparation}

The TUS detector production is at the conclusive phase. The technological prototype of the 7-module Fresnel mirror is produced and tested according to space qualification requirements as it is shown in Fig. 2. The TUS photo detector and electronics technological prototypes are also produced. Their tests are presently in progress.

A set of the program packages for TUS are producing including the event simulation with expected optical parameters, programs for on-board data processing and off-line physical analysis. A special attention is given to measurements of the Fresnel mirror system for an evaluation of systematic uncertainties in the data.

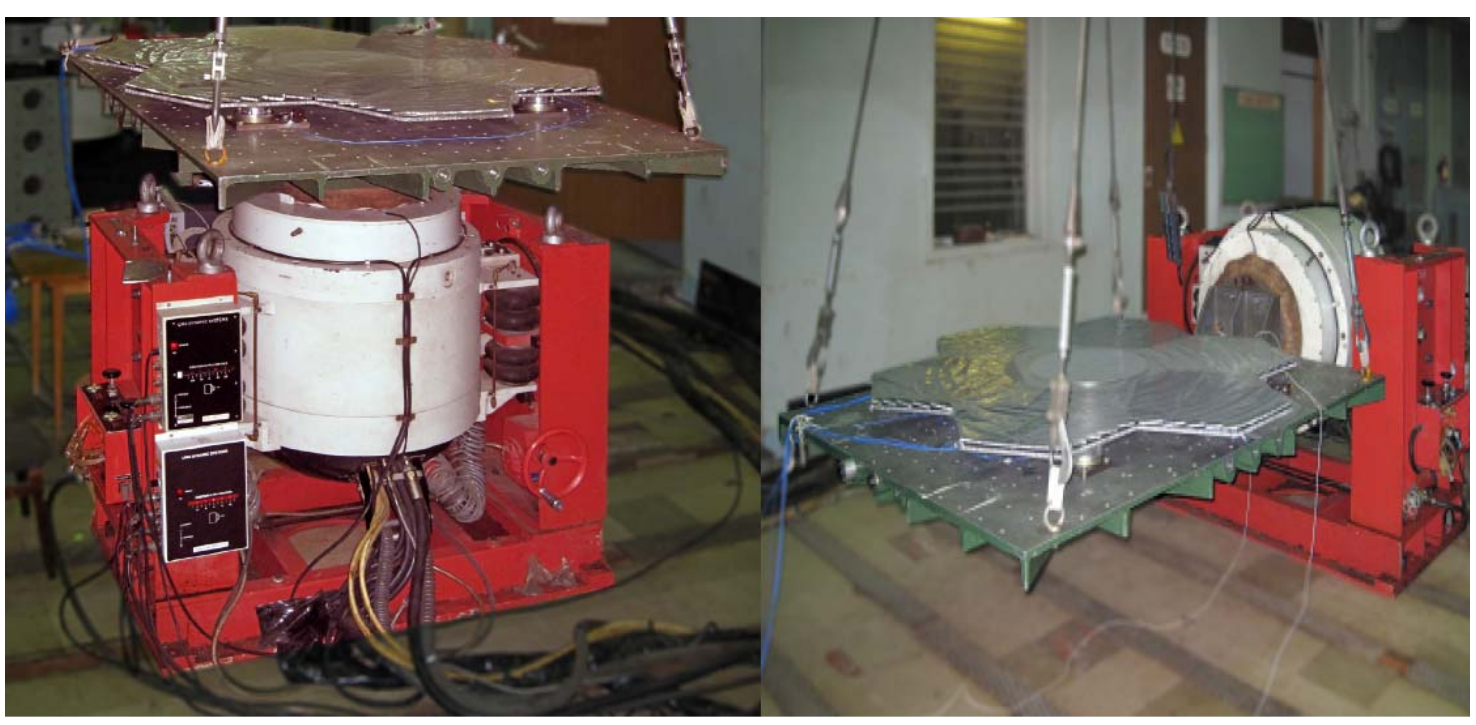

Fig.2. Space qualification tests of the technological Fresnel mirror

The flight TUS apparatus production is in preparation. The TUS mission is planned for operation at the end of 2011.

\section{References}

[1] V. Sadovnichi et al, The first results of the "Universitetsky-Tatiana" satellite. Cosmic Research, 45, (2007) 273-286.

[2] Y. Takahashi and the JEM-EUSO Collaboration, JEM-EUSO mission, 2009, New Journal of Physics, 11, 065009. 